International Journal of Pure and Applied Mathematics

Volume 89 No. $3 \quad$ 2013, 305-322

ISSN: 1311-8080 (printed version); ISSN: 1314-3395 (on-line version)

url: http://www.ijpam.eu

doi: http://dx.doi.org/10.12732/ijpam.v89i3.2

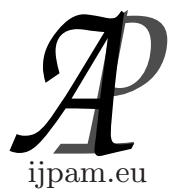

\title{
ON PSEUDO SPECTRA OF MATRICES
}

\author{
Mario Paul Ahues ${ }^{1 \S}$, Muzafar Fattah Hama² \\ ${ }^{1}$ Institut Camille Jordan, UMR 5208 \\ Université de Lyon \\ Université Jean Monnet \\ 23 Dr. Paul Michelon, 42023 Saint Etienne, FRANCE \\ ${ }^{2}$ University of Sulaimani New Camp \\ School of Science-Department of Mathematics \\ Tasluja street 1-Zone 501 \\ Sulaimani, Kurdistan Region, IRAQ
}

\begin{abstract}
We draw a parallel between the properties of the spectrum $\Lambda_{0}(A)$ and the pseudospectrum $\Lambda_{\epsilon}(A)$, for a square matrix $A$ and $\epsilon>0$. This paper presents several definitions and properties of the pseudospectrum as a function of the given matrix, $\epsilon>0$, and the underlying norm.
\end{abstract}

AMS Subject Classification: 65F15, 15A18

Key Words: geometric separation, spectrum, pseudospectrum, structured pseudospectrum

\section{Introduction}

Eigenvalue, latent roots, proper values, characteristic values are synonyms denoting a set of numbers which provide useful information about a matrix. Eigenvalues of matrices may be highly sensitive to perturbations (cf. [14], [15], [16]) and the spectral sensitivity analysis is more sophisticated than the linear system one.Classical results such as the Bauer-Fike theorem (cf. [10], [15]) have

Received: November 13, 2012

(c) 2013 Academic Publications, Ltd.

$\S$ Correspondence author url: www.acadpubl.eu 
been used in the literature to localize perturbated eigenvalues. Often a small perturbation may be sufficient for eigenvalues to change abruptly. The resulting eigenvalues may acquire characteristics which are radically different from those of the original ones. Two simple eigenvalues may change and become a multiple eigenvalue which may be defective as well. The notion of a successor was introduced in 1984, by Wilkinson (cf. [15]) for analyzing the evolution of perturbed eigenvalues when the magnitude of perturbation increases gradually. An important observation is the fact that individual eigenvalues of matrices have their own domain of influence in the complex plane which depend continuously on the magnitude of the perturbation. For a sufficiently small $\epsilon>0$, the $\epsilon$-domain of an eigenvalue $\lambda \in \Lambda_{0}(A)$ is the set of all complex numbers generated from $\lambda$ by all perturbations of $A$ of magnitude $\leq \epsilon$. Given $\epsilon>0$, a complex number $z$ is an $\epsilon$-successor of $\lambda \in \Lambda_{0}(A)$ if a perturbation of $A$ of magnitude $\leq \epsilon$ maps $\lambda$ into $z$. Thus each point in the $\epsilon$-domain of $\lambda$ is an $\epsilon$-successor of $\lambda$. As $\epsilon$ increases gradually the $\epsilon$-domain of an eigenvalue $\lambda$ intersects the $\epsilon$-domain of another neighbouring eigenvalue. We define the critical value of $\epsilon$ for which the $\epsilon$-domain of $\lambda$ intersects the $\epsilon$-domain of another neighbouring eigenvalue to be the geometric separation of $\lambda$ from the rest of $\Lambda_{0}(A)$ and denote it by $\operatorname{gsep}(\lambda)$, and the critical value of the magnitude of perturbation for which an eigenvalue $\lambda$ of a matrix $A$ equal to another eigenvalue is called the dissociation and is denoted by $\operatorname{diss}(\lambda)$. Evidently, $\operatorname{gsep}(\lambda) \leq \operatorname{diss}(\lambda)$. Although, it is not known whether $\operatorname{gsep}(\lambda)=\operatorname{diss}(\lambda)$ for arbitrary norms, but for specral norm this was proved by (cf. Bora $[5])$.

For practical purposes, it is the value of gsep that one really cares for; because as long as the magnitude of the perturbation remains whithin the safe distance of $\operatorname{gsep}(\lambda)$ each point in its domain of influence is a successor for the eigenvalue $\lambda$ and once this domain intersects a neighbouring domain these successors may lose their inherited properties. Therefore, the geometric separation of an eigenvalue is an accurate measure of its ill-conditioning. An eigenvalue may be well separated from the rest of the spectrum and yet its geometric separation may be small. In such a case, a small perturbation will cause a successor of the eigenvalue to coalesce with a successor of a neighbouring eigenvalue. This phenomenon is typically associated with a sensitive eigenvalue where a small perturbation dramatically increases its domain of influence. Thus an eigenvalue can safely be called ill-conditioned if and only if its geometric separation is small. The crux of the matter is the fact that the ill-conditioning of an eigenvalue is inversely proportional to its geometric separation (cf. [5]).

The implication of the existence of a common successor guaranteed by the gsep has been investigated in (cf. * [4]) leading to a complete solution of an 
open problem posed by Wilkinson (cf. [15], [16]) and localization theorems for eigenvalues can be effectively used to obtain upper and lower bounds of diss and effort have so far been directed towards achieving this (cf. [6]).

Due to various practical reasons, matrices may be known only up to a given accuracy. Even, when a matrix is know exactly, in finite precision computation it serves no better purpose than any other matrices which are indistinguishable from it up to the given accuracy. Therefore, if a matrix $A$ is known up to a given accuracy $\epsilon$, there exists a fundamental difficulty in undertaking spectral analysis of $A$. Under these circumstances, it becomes essential to define an appropriate notion of invertibility which takes into account the uncertainty present in $A$ up to the magnitude $\epsilon$. We thus introduce the notion of $\epsilon$-invertiblity of matrices. This leads naturally to the notion of the $\epsilon$-spectra of matrices.

We fix up notations which will be used throughout this paper. $\mathbb{R}$ denotes the real field, $\mathbb{C}$ the complex field. For both $\mathbb{F}=\mathbb{R}$ or $\mathbb{F}=\mathbb{C}, \mathbb{F}^{p \times q}$ denotes the linear space of matrices with $p$ rows and $q$ columns and we consider only submultiplicative norms. The $l^{p}$ and the Frobenious norms are defined by:

$$
\|x\|_{p}=\left(\sum_{i=1}^{n}|x(i)|^{p}\right)^{\frac{1}{p}}, 1 \leq p<\infty, \quad\|x\|_{\infty}=\max _{1 \leq i \leq n}|x(i)|,
$$

and

$$
\|A\|_{F}=\sqrt{\sum_{i, j=1}^{n}|A(i, j)|^{2}}
$$

respectively.

We use the notation $\mathcal{B}(x, \epsilon)$, (resp. $\mathcal{B}[x, \epsilon]$ ) for open (resp. closed) ball which are defined in any normed space $X$ as

$$
\mathcal{B}(x, \epsilon)=\{y \in X:\|y-x\|<\epsilon\},
$$

and

$$
\mathcal{B}[x, \epsilon]=\{y \in X:\|y-x\| \leq \epsilon\} .
$$

We say that $\|\cdot\|$ has the maximum property iff

$$
\left\|\operatorname{diag}\left(A_{1}, A_{2}\right)\right\|=\max \left(\left\|A_{1}\right\|,\left\|A_{2}\right\|\right) .
$$

By knowing $A$ within a tolerance $\epsilon$ we mean that $A$ is indistinguishable from any matrix in the ball $\mathcal{B}[A, \epsilon]$. 
Definition 1. Let $U$ be an open connected subset of $\mathbb{C}$. A function $f$ : $U \rightarrow \mathbb{R} \cup\{-\infty\}$ is said to be subharmonic on $U$ iff it is upper semicontinuous on $U$ and satisfies the inequality

$$
f\left(z_{0}\right) \leq \frac{1}{2 \pi} \int_{0}^{2 \pi} f\left(z_{0}+r e^{i \theta}\right) d \theta
$$

for all closed disks $\mathcal{B}\left[z_{0}, r\right] \subseteq U$.

The motivation for the concept pseudospectra comes from numerical eigenvalue computations. It is well known that in problems involving nonnormal matrices, eigenvalue computations are ill-conditioned.

\section{The $\epsilon$-Spectra of Matrices}

The notion of the $\epsilon$-spectrum of a matrix $A \in \mathbb{F}^{n \times n}$, as far as we know, was first introduced by Varah (cf. [13]) in 1979:

$$
\Lambda_{\epsilon}(A)=\left\{z \in \mathbb{C}: \sigma_{\min }(A-z I) \leq \epsilon\right\} .
$$

The following notions will be use useful in the sequel.

Definition 2. The complement in $\mathbb{C}$ of the spectrum of $A \in \mathbb{C}$ is called the resolvent set of $A$ and will be denoted by $\operatorname{re}(A)$. For $z \in \operatorname{re}(A)$, the matrix

$$
R(A, z)=(A-z I)^{-1}
$$

is called the resolvent of $A$ at $z$.

Subsequently, this definition has been modified and the set $\Lambda_{\epsilon}(A)$ has come to be widely refered to as the $\epsilon$-pseudospectrum of $A$ (cf. [11], [12]) redefined by:

$$
\Lambda_{\epsilon}(A)=\left\{z \in \mathbb{C}:\|R(A, z)\| \geq \frac{1}{\epsilon}\right\} .
$$

The notion of a successor of an eigenvalue was introduced by Wilkinson: A complex number $z$ is a successor of an eigenvalue $\lambda$ if a perturbation maps $\lambda$ into $z$. This definition, however, does not exclude the possibility for $z$ of being a successor of another eigenvalue $\mu \in \Lambda_{0}(A)$. This happens when the domains of influence of $\lambda$ and $\mu$ intersect as the magnitude of perturbation increases gradually to $\epsilon$. Thus we could define the $\epsilon$-spectrum of $A$ to be the set of all $\epsilon$-successors of its eigenvalues. 
Definition 3. Let $\epsilon \geq 0$ and $A \in \mathbb{F}^{n \times n}$. $A$ is said to be $\epsilon$-invertible iff each $B \in \mathcal{B}[A, \epsilon]$ is invertible. $A$ is said to be $\epsilon$-singular iff $A$ is not $\epsilon$-invertible.

Thus we have another definition of $\Lambda_{\epsilon}(A)$ which is the set of all complex numbers $z$ such that $A-z I$ is $\epsilon$-singular. Also, we have another definition of $\Lambda_{\epsilon}(A)$ that uses the concept of separation of $z$ from $A$ :

$$
\operatorname{sep}(z, A)=\inf \left\{\|E\|: z \in \Lambda_{0}(A+E)\right\} .
$$

Evidently, if $z$ is $\epsilon$-successor of $\lambda \in \Lambda_{0}(A)$ then $\operatorname{sep}(z, A) \leq \epsilon$. We denote by $\operatorname{sep}_{p}(z, A)\left(\operatorname{resp} . \operatorname{sep}_{F}(z, A)\right)$ the separation of $z$ from $A$ with respect to the $l^{p}$ (resp. Frobenius) norm. The function $\operatorname{sep}(z, A)$ has similar properties as those of the smallest singular value of $A-\lambda I$ and plays a crucial role when analyzing the sensitivity of eigenvalues and eigendecompositions of $A$.

The standard compactness argument shows that the infimum in the definition of $\operatorname{sep}(z, A)$ is actually the minimum. Indeed, the definition of $\operatorname{sep}(z, A)$ shows that there exists a sequence $\left(E_{n}\right)_{n \geq 1}$ such that $\left\|E_{n}\right\| \rightarrow \operatorname{sep}(z, A)$ and $z \in \Lambda_{0}\left(A+E_{n}\right)$. Now the result follows from the continuity of the spectrum of $A$ and the fact that $\left(E_{n}\right)_{n \geq 1}$ has a convergent subsequence converging to $E$ such that $\|E\|=\operatorname{sep}(z, A)$.

Evidently $\operatorname{sep}(z, A)=\operatorname{sep}(0, A-z I)$ and for nonzero $\alpha \in \mathbb{C}, \operatorname{sep}(\alpha z, A)=$ $|\alpha| \operatorname{sep}\left(z, \frac{A}{\alpha}\right)$ and $\operatorname{sep}(z, \alpha A)=|\alpha| \operatorname{sep}\left(\frac{z}{\alpha}, A\right)$.

Proposition 4. Let $\epsilon>0$ and $A \in \mathbb{C}^{n \times n}$. $A$ is $\epsilon$-invertible iff $\operatorname{sep}(0, A)>\epsilon$.

Proof.

$(\Longrightarrow)$ Suppose that $A$ is $\epsilon$-invertible. If $\operatorname{sep}(0, A) \leq \epsilon$, then $\inf \{\|E\|: 0 \in$ $\left.\Lambda_{0}(A+E)\right\} \leq \epsilon$. Thus, $\operatorname{det}(A+E)=0$. This means that $B=A+E$ is not invertible and $B \in \mathcal{B}[A, \epsilon]$. Since this is absurd, $\operatorname{sep}(0, A)>\epsilon$.

$(\Longleftarrow)$ Suppose that $\inf \left\{\|E\|: 0 \in \Lambda_{0}(A+E)\right\}>\epsilon$. We show that A is $\epsilon$-invertible. If not, there exists $B \in \mathcal{B}[A, \epsilon]$ such that $B$ is not invertible i.e. $\operatorname{det}(B)=0$. Thus we could define $E=B-A$ such that $\|E\| \leq \epsilon$ and this is absurd.

Theorem 5. The following holds:

1. For the subordinated norm on $\mathbb{F}^{n \times n}$,

$$
\operatorname{sep}(z, A)=\min \{\|A x-z x\|:\|x\|=1\}=\frac{1}{\|R(A, z)\|} \text { for } z \in \operatorname{re}(A) .
$$

2. For $z \in \mathbb{C}, \operatorname{sep}_{2}(z, A)=\operatorname{sep}_{F}(z, A)=\sigma_{\min }(A-z I)$. 
3. Let $\kappa(X)=\|X\|\left\|X^{-1}\right\|$. Then

$$
\frac{\operatorname{sep}\left(z, X^{-1} A X\right)}{\kappa(X)} \leq \operatorname{sep}(z, A) \leq \kappa(X) \operatorname{sep}\left(z, X^{-1} A X\right)
$$

Proof. See [3].

Proposition 6. For the 2-norm in $\mathbb{C}^{n \times 1}$, the subordinated one in $\mathbb{C}^{n \times n}$ and $\epsilon>0$ :

1. $\Lambda_{\epsilon}(A)=\left\{z \in \mathbb{C}: \sigma_{\min }(A-z I) \leq \epsilon\right\}$,

2. $\Lambda_{\epsilon}(A)=\{z \in \mathbb{C}: \min \{\|(A-z I) x\|:\|x\|=1\} \leq \epsilon\}$,

3. $\Lambda_{\epsilon}(A)=\left\{z \in \mathbb{C}:\|R(A, z)\| \geq \epsilon^{-1}\right\}$,

4. $\Lambda_{\epsilon}(A)=\left\{z \in \mathbb{C}: \exists E \in \mathbb{C}^{n \times n},\|E\| \leq \epsilon, z \in \Lambda_{0}(A+E)\right\}$,

5. $\Lambda_{\epsilon}(A)$ is equal to the set of all $\epsilon$-successors of the eigenvalues of $A$,

6. $\Lambda_{\epsilon}(A)$ is equal to the set of all complex numbers $z$ such that $A-z I$ is $\epsilon$-singular,

7. $\Lambda_{\epsilon}(A)=\left\{z \in \mathbb{C}: \operatorname{sep}_{2}(z, A) \leq \epsilon\right\}$,

8. $\Lambda_{\epsilon}(A)=\bigcup_{\|E\| \leq \epsilon} \Lambda_{0}(A+E)$,

Proof. The first three characterizations follow from the identity $\sigma_{\min }(A-$ $z I)=\|R(A, z)\|^{-1}=\min \{\|(A-z I) x\|:\|x\|=1\}$ and theorem (5).

$(3) \Longrightarrow(4)$ : We interpret $E$ as a perturbation of $A-z I$ into a singular matrix that is, $z$ is the eigenvalue of $\Lambda_{0}(A+E)$ and we use the result of Eckart and Young $[7$, theorem (6.5)]: The distance to singularity in the 2-norm equals the reciprocal of the norm of the inverse.

$(4) \Longrightarrow(5)$ : Let $z \in \Lambda_{\epsilon}(A)$. Then there exists $E \in \mathbb{C}^{n \times n}$ such that $\|E\| \leq \epsilon$ and $z \in \Lambda_{0}(A+E)$. Thus $z$ is a successor of an eigenvalue of $\Lambda_{0}(A)$.

$(5) \Longrightarrow(6)$ : If $z$ is a successor of an eigenvalue $\lambda \in \Lambda_{0}(A)$ then there exists $E$ such that $\|E\| \leq \epsilon$ and $\left.z \in \Lambda_{0}(A+E)\right)$. Hence $\operatorname{det}(A+E-z I)=0$. Set $B=(A+E-z I)$. $B$ is not invertible and $\|(A-z I)-B\|=\|E\| \leq \epsilon$. Hence we have found $B \in \mathcal{B}(A-z I, \epsilon)$ which is not $\epsilon$-invertible. Therefore $A-z I$ is $\epsilon$-singular.

$(6) \Longrightarrow(7)$ : From the properties of sep in [3] we have $\operatorname{sep}(0, A-z I)=$ $\operatorname{sep}(z, A)$, and from Proposition (4), $A-z I$ is $\epsilon$-singular iff $A-z I$ is not $\epsilon$ invertible iff $\operatorname{sep}(0, A-z I) \leq \epsilon$ iff $\operatorname{sep}(z, A) \leq \epsilon$. 
$(7) \Longrightarrow(8):$ Let $z \in \Lambda_{\epsilon}(A)$ and $\operatorname{sep}_{2}(z, A) \leq \epsilon \cdot \inf \left\{\|E\|, z \in \Lambda_{0}(A+E)\right\} \leq \epsilon$ implies $z \in \bigcup_{\|E\| \leq \epsilon} \Lambda_{0}(A+E)$.

$(8) \Longrightarrow(1)$ : See Theorem (5).

Remark 7. Also for the Frobenius norm,

$$
\Lambda_{\epsilon}(A)=\left\{z \in \mathbb{C}: \operatorname{sep}_{F}(z, A) \leq \epsilon\right\} .
$$

Proposition 8. $\Lambda_{\epsilon}(A)$ is compact and does not have isolated points. Thus $\Lambda_{\epsilon}(A)$ consists of nontrivial components.

Proof. See [3].

Proposition 9. For a matrix norm the function $z \mapsto\|R(A, z)\|$ is nonconstant on open subsets of $\operatorname{re}(A)$.

Proof. Suppose that there exists $c \neq 0$ and an open subset $U$ of $\operatorname{re}(A)$ such that $\|R(A, z)\|=c$ for all $z \in U$. By theorem $(5) \operatorname{sep}(z, A)=\frac{1}{c}$ for all $z \in U \subseteq \Lambda_{0}(A)^{c}, \operatorname{sep}(z, A)=\|R(A, z)\|^{-1}$. Let $w \in U$. There exists $E \in \mathbb{C}^{n \times n}$ such that $\|E\|=\frac{1}{c}$ and $w \in \Lambda_{0}(A+E)$. Suppose that $\left(E_{n}\right)_{n>1}$ is a sequence in $\mathbb{C}^{n \times n}$ such that $E_{n} \rightarrow E$ and $\left\|E_{n}\right\|<\frac{1}{c}$ for $n \in \mathbb{N}$. Consequently, $A+E_{n} \rightarrow A+E$ but from [2] $\Lambda_{0}(A+E)$ has the property of lower semi-continuity at each point, i.e. there exists $n_{0}$ such that $\Lambda_{0}\left(A+E_{n}\right) \cap U \neq \emptyset$ for all $n \geq n_{0}$ that is, there exists $z_{n} \in \mathbb{C}$ such that $z_{n} \in \Lambda_{0}\left(A+E_{n}\right) \cap U$ for all $n \geq n_{0}$. But this is implies $\operatorname{sep}\left(z_{n}, A\right)<\frac{1}{c}$ and $z_{n} \in U$, which is contradictory.

Another proof can be found in [5].

\section{Pseudospectrum and Structured Perturbations}

In this section $\|\cdot\|$ denotes the $\ell^{2}$-norm for vectors and the subordinated one for matrices. The definition of pseudospectra of $A$ is taken as

$$
\Lambda_{\epsilon}(A)=\left\{z \in \mathbb{C}: \exists E \in \mathbb{F}^{n \times n},\|E\| \leq \epsilon, z \in \Lambda_{0}(A+E)\right\} .
$$

For structured matrices it seems also resonable to look at the structured pseudopectra by limiting the perturbations to some structure. If instead of $E$ we choose only matrices $E \in \mathbb{F}^{n \times n}$ having the same properties as $A$ we reduce $\Lambda_{\epsilon}(A)$ to the so-called structured pseudospectrum. It is denoted by $\Lambda_{\epsilon}^{\mathbb{S}}(A)$ :

$$
\Lambda_{\epsilon}^{\mathbb{S}}(A)=\left\{z \in \mathbb{F}: \exists E \in \mathbb{S} \cap \mathbb{F}^{n \times n},\|E\| \leq \epsilon, z \in \Lambda_{0}(A+E)\right\},
$$


where $\mathbb{S}$ denotes a family structured matrices. We are interested in the following choices:

\begin{tabular}{|c|c|}
\hline Family $\mathbb{S}$ & Symbol \\
\hline symmetric & $\mathrm{Sy}$ \\
\hline Hermitian & $\mathrm{He}$ \\
\hline skewsymmetric & $\mathrm{Ss}$ \\
\hline skewHermitian & $\mathrm{Sh}$ \\
\hline persymmetric & $\mathrm{Ps}$ \\
\hline Toeplitz & $\mathrm{To}$ \\
\hline symmetric Toeplitz & $\mathrm{St}$ \\
\hline Hankel & $\mathrm{Ha}$ \\
\hline persymmetric Hankel & $\mathrm{Ph}$ \\
\hline circulant & $\mathrm{Ci}$ \\
\hline
\end{tabular}

S. M. Rump has characterized $\Lambda_{\epsilon}^{\mathbb{S}}(A)$ for most of the structures in (1). In fact, for many of them he showed that $\Lambda_{\epsilon}(A)=\Lambda_{\epsilon}^{\mathbb{S}}(A)$. However, he remarked that for infinite Toeplitz matrices the equality $\Lambda_{\epsilon}^{\mathbb{S}}(A)=\Lambda_{\epsilon}(A)$ is in general not true (cf. [9]).

\subsection{Properties of $\Lambda_{\epsilon}(A)$ Relative to Matrix $A$}

The following results present some basic properties of $\Lambda_{\epsilon}(A)$ relative to the subordinated norm. Let

$$
\omega_{\epsilon}(A)=\{z \in \mathbb{C}: \operatorname{sep}(z, A)<\epsilon\} .
$$

Theorem 10. The pseudospectrum $\Lambda_{\epsilon}(A)$ relative to the subordinated norm satisfies:

1. $\Lambda_{\epsilon}(A)$ is the closure of $\omega_{\epsilon}(A)$, and

$$
\partial \Lambda_{\epsilon}(A) \subseteq \partial \omega_{\epsilon}(A)=\left\{z \in \operatorname{re}(A):\|R(A, z)\|=\epsilon^{-1}\right\} .
$$

2. Each component of $\Lambda_{\epsilon}(A)$ contains at least one eigenvalue of $A$ in its interior.

We recall that, for any nonsingular matrix $V \in \mathbb{F}^{n \times n}, \Lambda_{0}\left(V^{-1} A V\right)=\Lambda_{0}(A)$. Also, for $A=\operatorname{diag}\left(A_{1}, A_{2}\right) \in \mathbb{F}^{n \times n}, \Lambda_{0}(A)=\Lambda_{0}\left(A_{1}\right) \cup \Lambda_{0}\left(A_{2}\right)$. In terms of pseudospectra, these situations change as follows: 
Theorem 11. If $V$ is nonsingular matrix, then, for any submultiplicative norm, $\Lambda_{\epsilon}(A) \subseteq \Lambda_{\kappa(V) \epsilon}\left(V^{-1} A V\right)$ and $\Lambda_{\epsilon}\left(V^{-1} A V\right) \subseteq \Lambda_{\kappa(V) \epsilon}(A)$.

Proof. See [5].

Corollary 12. If $V$ is nonsingular matrix such that $\kappa(V)=1$ then, for any submultiplicative norm, $\Lambda_{\epsilon}(A)=\Lambda_{\epsilon}\left(V^{-1} A V\right)$.

Remark 13. Let $A=\operatorname{diag}\left(A_{1}, A_{2}\right)$, where

$$
A_{1}=\left[\begin{array}{cc}
1 & 0 \\
0 & -1
\end{array}\right], \quad A_{2}=\left[\begin{array}{cc}
i & 0 \\
0 & -i
\end{array}\right] .
$$

Take the Frobenius norm on $\mathbb{C}^{n \times n}$. Set $\epsilon=\frac{1}{2}$. For $z=0$

$$
\left\|(A-z I)^{-1}\right\|_{F}=\left\|\left[\begin{array}{cccc}
1 & 0 & 0 & 0 \\
0 & -1 & 0 & 0 \\
0 & 0 & -i & 0 \\
0 & 0 & 0 & i
\end{array}\right]\right\|_{F}=2 \geq \epsilon^{-1}
$$

that is, $0 \in \Lambda_{\epsilon}(A)$. But

$$
\begin{aligned}
\left\|\left(A_{1}-z I_{1}\right)^{-1}\right\|_{F} & =\left\|\left[\begin{array}{cc}
1 & 0 \\
0 & -1
\end{array}\right]\right\|_{F} \\
& =\sqrt{2} \leq \epsilon^{-1}, \\
\left\|\left(A_{2}-z I_{2}\right)^{-1}\right\|_{F} & =\left\|\left[\begin{array}{cc}
-i & 0 \\
0 & i
\end{array}\right]\right\|_{F} \\
& =\sqrt{2} \leq \epsilon^{-1} .
\end{aligned}
$$

So $0 \notin\left(\Lambda_{\epsilon}\left(A_{1}\right) \cup \Lambda_{\epsilon}\left(A_{2}\right)\right)$.

Theorem 14. For any subordinated norm having the maximum property,

$$
\Lambda_{\epsilon}\left(\operatorname{diag}\left(A_{1}, A_{2}\right)\right)=\Lambda_{\epsilon}\left(A_{1}\right) \cup \Lambda_{\epsilon}\left(A_{2}\right) .
$$

Proof. See [5].

Proposition 15. Let $A \in \mathbb{C}^{n \times n}$ be a normal matrix, then

$$
\Lambda_{\epsilon}(A)=\left\{z \in \mathbb{C}: \operatorname{dist}\left(z, \Lambda_{0}(A) \leq \epsilon\right\}=\bigcup_{\lambda \in \Lambda_{0}(A)} \mathcal{B}[\lambda, \epsilon] .\right.
$$


Proof. Since $A$ is normal, there exists a unitary matrix $Q$ such that $D=$ $Q^{*} A Q$ is a diagonal matrix. Let $\lambda_{i}=D(i, i)$. Since

$$
\|R(A, z)\|=\frac{1}{\max _{i \in \llbracket 1, n \rrbracket}\left|z-\lambda_{i}\right|},
$$

the result follows.

Theorem 16. Let $A, B \in \mathbb{F}^{n \times n}$ have eigenvalues $\alpha_{1}, \alpha_{2}, \ldots, \alpha_{n}$ and $\beta_{1}, \beta_{2}, \ldots, \beta_{n}$, respectively. If $A$ and $B$ commute, there exists a pemutation $i_{1}, i_{2}, \ldots, i_{n}$ of the indexes $1,2, \ldots, n$ such that the eigenvalues of $A+B$ are $\alpha_{1}+\beta_{i_{1}}, \alpha_{2}+\beta_{i_{2}}, \ldots, \alpha_{n}+\beta_{i_{n}}$. In particular, $\Lambda_{0}(A+B) \subseteq \Lambda_{0}(A)+\Lambda_{0}(B)$.

Proof. See [8].

The above theorem gives:

Corollary 17. Let $A \in \mathbb{F}^{n \times n}$. For any $\epsilon>0$ and matrix $E \in \mathbb{F}^{n \times n}$ which commutes with $A$ such that $\|E\| \leq \epsilon$,

$$
\Lambda_{\epsilon}(A) \subseteq \Lambda_{0}(A)+\bigcup_{\substack{\|E\| \leq \epsilon \\ A E=E A}} \Lambda_{0}(E) .
$$

Remark 18. In Corollary 17, it is necessary that $A$ and $E$ commute. Let

$$
A=\left[\begin{array}{ll}
0 & 1 \\
0 & 0
\end{array}\right], \quad E=\left[\begin{array}{ll}
0 & 0 \\
1 & 0
\end{array}\right], \epsilon=1,
$$

and take the $\ell^{2}$-norm. Then $A E \neq E A$ and $\Lambda_{1}(A)$ is equal to $\{-1,1\}$ but $\Lambda_{0}(A)=\{0\}=\Lambda_{0}(E)$.

We recall that if $\Omega$ is an open set in $\mathbb{C}$ containing $\Lambda_{0}(A), f: \Omega \rightarrow \mathbb{C}$ an analytic function on $\Omega$ and $C$ a Jordan closed curve in $\Omega$ enclosing $\Lambda_{0}(A)$, the operator $f(A)$ is defined by

$$
f(A)=-\frac{1}{2 \pi i} \int_{C} f(z) R(A, z) d z .
$$

Theorem 19. Let $A \in \mathbb{C}^{n \times n}, \Omega$ be an open set in $\mathbb{C}$ which contains $\Lambda_{0}(A)$, and $f: \Omega \rightarrow \mathbb{C}$ an analytic function on $\Omega$.

$$
f\left(\Lambda_{0}(A)\right)=\Lambda_{0}(f(A))
$$


Proof. See [1].

Theorem 20. Let $\left.\left.A \in \mathbb{C}^{n \times n}, \epsilon \in\right] 0,1\right], \Omega$ be an open set in $\mathbb{C}$ containing the closed ball centered at 0 and with radius $r=2+\|A\|, f: \Omega \rightarrow \mathbb{C}$ an analytic function on $\Omega$. The following inclusion holds:

$$
f\left(\Lambda_{\epsilon}(A)\right) \subseteq \Lambda_{\eta}(f(A))
$$

where

$$
\eta=\epsilon r \max _{|z|=r}|f(z)|
$$

Proof. If $\mu \in f\left(\Lambda_{\epsilon}(A)\right)$ then there exists $\lambda \in \Lambda_{\epsilon}(A)$ such that $\mu=f(\lambda)$. Hence, for some $E$ such that $\|E\| \leq \epsilon$,

$$
\begin{aligned}
\mu & =f(\lambda) \in f\left(\Lambda_{0}(A+E)\right) \\
& =\Lambda_{0}(f(A+E)) \\
& =\Lambda_{0}\left(f(A)+\int_{0}^{1} D f(A+t E) E d t\right) \\
& =\Lambda_{0}(f(A)+F),
\end{aligned}
$$

where

$$
F=f(A+E)-f(A),
$$

and $\operatorname{Df}(X)$ is the unique linear operator from $\mathbb{C}^{n \times n}$ into $\mathbb{C}^{n \times n}$ such that

$$
\lim _{E \rightarrow O} \frac{f(X+E)-f(X)-D f(X) E}{\|E\|}=O .
$$

Let $C$ be the circumference of centre 0 and radius $r$. Then $C$ encloses $\Lambda_{\epsilon}(A)$. For $E$ such that $\|E\| \leq \epsilon$,

$$
\begin{aligned}
f(X+E)-f(X) & =-\frac{1}{2 \pi i} \int_{C^{+}} f(z)[R(X+E, z)-R(X, z)] d z \\
& =\frac{1}{2 \pi i} \int_{C^{+}} f(z)[R(X, z) E R(X, z)] d z+O\left(E^{2}\right) .
\end{aligned}
$$

We conclude that

$$
D f(X) E=\frac{1}{2 \pi i} \int_{C^{+}} f(z)[R(X, z) E R(X, z)] d z
$$

Thus we have $\mu \in \Lambda_{0}(f(A)+F)$ where

$$
\|F\| \leq \max _{0 \leq t \leq 1}\left\|\frac{1}{2 \pi i} \int_{C^{+}} f(z)[R(A+t E, z) E R(A+t E, z)] d z\right\| .
$$


But, for $z \in C$ and $0 \leq t \leq 1$,

$$
\|R(A+t E, z)\| \leq \frac{1}{|z|-\|A+t E\|} \leq \frac{1}{r-\|A\|+\|E\|} \leq 1
$$

Hence

$$
\|F\| \leq \eta=\epsilon r \max _{|z|=r}|f(z)|
$$

which ends the proof.

We recall that, for all $A \in \mathbb{C}^{n \times n}, \Lambda_{0}(A)^{*}=\Lambda_{0}\left(A^{*}\right)$ and hence, if $A=A^{*}$ then $\Lambda_{0}(A)^{*}=\Lambda_{0}\left(A^{*}\right)=\Lambda_{0}\left(A^{*}\right)^{*}=\Lambda_{0}(A)$.

Proposition 21. For $A \in \mathbb{R}^{n \times n}$ and in the $\ell^{2}$-norm,

$$
\Lambda_{\epsilon, \mathbb{R}}(A)=\Lambda_{\epsilon, \mathbb{R}}\left(A^{*}\right)=\Lambda_{\epsilon, \mathbb{R}}(A)^{*} .
$$

Proof. Let $z \in \Lambda_{\epsilon}(A)$. There exists $E \in \mathbb{R}^{n \times n}$ such that $\|E\| \leq \epsilon$ and

$$
z \in \Lambda_{0}(A+E) \text { implies } z^{*} \in \Lambda_{0}\left(A^{*}+E^{*}\right) .
$$

Now $\left\|E^{*}\right\|=\|E\| \leq \epsilon$ and since $A, E$ are in $\mathbb{R}^{n \times n}, z \in \Lambda_{0}\left(A^{*}+E^{*}\right)$.

Conversely, suppose that $z \in \Lambda_{\epsilon, \mathbb{R}}\left(A^{*}\right)$. There exists $E \in \mathbb{R}^{n \times n}$ such that $\|E\| \leq \epsilon$ and $z \in \Lambda_{0}\left(A^{*}+E\right)$. Since $A \in \mathbb{R}^{n \times n}$ and $\left\|E^{*}\right\|=\|E\| \leq \epsilon$, then $z^{*} \in \Lambda_{0}\left(A^{*}+E\right)$ and $z \in \Lambda_{0}\left(A+E^{*}\right)$. Consequently $z \in \Lambda_{\epsilon, \mathbb{R}}\left(A^{*}\right)$ and $\Lambda_{\epsilon, \mathbb{R}}\left(A^{*}\right)=$ $\Lambda_{\epsilon, \mathbb{R}}(A)$.

Now, let $z \in \Lambda_{\epsilon, \mathbb{R}}(A)$. There exists $E \in \mathbb{R}^{n \times n}$ such that $\|E\| \leq \epsilon$ and $z \in \Lambda_{0}(A+E)$. Since $A+E \in \mathbb{R}^{n \times n}, z^{*} \in \Lambda_{0}(A+E)$. Hence $z \in \Lambda_{\epsilon, \mathbb{R}}(A)^{*}$.

Conversely, let $z \in \Lambda_{\epsilon, \mathbb{R}}(A)^{*}$ that is, $z^{*} \in \Lambda_{\epsilon}(A)$. There exists $E \in \mathbb{R}^{n \times n}$ such that $\|E\| \leq \epsilon$ and $z^{*} \in \Lambda_{0}(A+E)$. Since $A+E \in \mathbb{R}^{n \times n}, z \in \Lambda_{\epsilon}(A)$. Consequently, $\Lambda_{\epsilon, \mathbb{R}}\left(A^{*}\right)=\Lambda_{\epsilon, \mathbb{R}}(A)^{*}=\Lambda_{\epsilon, \mathbb{R}}(A)$.

Proposition 22. For $A \in \mathbb{C}^{n \times n}$ and in the $\ell^{2}$-norm,

$$
\Lambda_{\epsilon}(A)^{*}=\Lambda_{\epsilon}(A)
$$

Proof. Let $z \in \Lambda_{\epsilon}(A)^{*}$. There exists $E \in \mathbb{C}^{n \times n}$ such that $\|E\| \leq \epsilon$ and $z^{*} \in \Lambda_{0}(A+E)$. Hence $z \in \Lambda_{0}\left(A^{*}+E^{*}\right)$. But $\left\|E^{*}\right\|=\|E\| \leq \epsilon$, so $z \in \Lambda_{\epsilon}\left(A^{*}\right)$.

Conversely, suppose that $z \in \Lambda_{\epsilon}\left(A^{*}\right)$. There exists $E \in \mathbb{C}^{n \times n}$ such that $\|E\| \leq \epsilon$ and $z^{*} \in \Lambda_{0}(A+E)=\Lambda_{0}(A+E)^{*}$. But $\left\|E^{*}\right\|=\|E\| \leq \epsilon$. Hence $z \in \Lambda_{\epsilon}(A)^{*}$. Consequently, $\Lambda_{\epsilon}(A)^{*}=\Lambda_{\epsilon}\left(A^{*}\right)$. 
Corollary 23. Let $A \in \mathbb{C}^{n \times n}$ be such that $A^{*}=A$ and consider the $\ell^{2}$-norm. Then

$$
\Lambda_{\epsilon}(A)=\Lambda_{\epsilon}(A)^{*}=\Lambda_{\epsilon}\left(A^{*}\right)=\Lambda_{\epsilon}\left(A^{*}\right)^{*} .
$$

Remark 24. The converse of Corollary 23 is not true. Let

$$
A=\left[\begin{array}{ll}
0 & i \\
0 & 0
\end{array}\right] \neq A^{*}=\left[\begin{array}{cc}
0 & 0 \\
-i & 0
\end{array}\right]
$$

We see that $\Lambda_{\epsilon}(A)=\Lambda_{\epsilon}(A)^{*}=\Lambda_{\epsilon}\left(A^{*}\right)=\Lambda_{\epsilon}\left(A^{*}\right)^{*}$. In fact, $z \in \Lambda_{\epsilon}(A)=\{z \in$ $\left.\mathbb{C}:\left\|(A-z I)^{-1}\right\| \geq \frac{1}{\epsilon}\right\}$,

$$
\left\|(A-z I)^{-1}\right\|=\left\|\left(\begin{array}{cc}
-z & i \\
0 & -z
\end{array}\right)^{-1}\right\|=\frac{1}{|z|^{2}} \sqrt{\rho\left(\begin{array}{cc}
|z|^{2} & i \bar{z} \\
-i z & |z|^{2}+1
\end{array}\right)},
$$

and

$$
\begin{aligned}
\rho_{1} & =\rho\left(\begin{array}{cc}
|z|^{2} & i \bar{z} \\
-i z & |z|^{2}+1
\end{array}\right) \\
& =\max \left\{|\lambda|:\left(|z|^{2}-\lambda\right)\left(|z|^{2}+1-\lambda\right)-|z|^{2}=0\right\}
\end{aligned}
$$

Now if $z \in \Lambda_{\epsilon}(A)^{*}=\left\{z^{*} \in \mathbb{C}:\left\|(A-\bar{z} I)^{-1}\right\| \geq \frac{1}{\epsilon}\right\}$,

$$
\left\|(A-\bar{z} I)^{-1}\right\|=\left\|\left(\begin{array}{cc}
-\bar{z} & i \\
0 & -\bar{z}
\end{array}\right)^{-1}\right\|=\frac{1}{|z|^{2}} \sqrt{\rho\left(\begin{array}{cc}
|z|^{2} & i z \\
-i \bar{z} & |z|^{2}+1
\end{array}\right)},
$$

and

$$
\rho_{2}=\rho\left(\begin{array}{cc}
|z|^{2} & i z \\
-i \bar{z} & |z|^{2}+1
\end{array}\right)=\max \left\{|\lambda|:\left(|z|^{2}-\lambda\right)\left(|z|^{2}+1-\lambda\right)-|z|^{2}=0\right\} .
$$

Also if $z \in \Lambda_{\epsilon}\left(A^{*}\right)=\left\{z \in \mathbb{C}:\left\|\left(A^{*}-z I\right)^{-1}\right\| \geq \frac{1}{\epsilon}\right\}$,

$$
\left\|\left(A^{*}-z I\right)^{-1}\right\|=\left\|\left(\begin{array}{cc}
-z & 0 \\
-i & -z
\end{array}\right)^{-1}\right\|=\frac{1}{|z|^{2}} \sqrt{\rho\left(\begin{array}{cc}
|z|^{2}+1 & i z \\
-i \bar{z} & |z|^{2}
\end{array}\right)}
$$

and

$$
\rho_{3}=\rho\left(\begin{array}{cc}
|z|^{2}+1 & i z \\
-i \bar{z} & |z|^{2}
\end{array}\right)=\max \left\{|\lambda|:\left(|z|^{2}+1-\lambda\right)\left(|z|^{2}-\lambda\right)-|z|^{2}=0\right\} .
$$


Finally, if $z \in \Lambda_{\epsilon}\left(A^{*}\right)^{*}=\left\{z^{*} \in \mathbb{C}:\left\|\left(A^{*}-\bar{z} I\right)^{-1}\right\| \geq \frac{1}{\epsilon}\right\}$,

$$
\left\|\left(A^{*}-\bar{z} I\right)^{-1}\right\|=\left\|\left(\begin{array}{cc}
-\bar{z} & 0 \\
-i & -\bar{z}
\end{array}\right)^{-1}\right\|=\frac{1}{|z|^{2}} \sqrt{\rho\left(\begin{array}{cc}
|z|^{2}+1 & i z \\
-i \bar{z} & |z|^{2}
\end{array}\right)}
$$

and

$$
\rho_{4}=\rho\left(\begin{array}{cc}
|z|^{2}+1 & i z \\
-i \bar{z} & |z|^{2}
\end{array}\right)=\max \left\{|\lambda|:\left(|z|^{2}-\lambda\right)\left(|z|^{2}+1-\lambda\right)-|z|^{2}=0\right\}
$$

We observe that the $\rho_{1}=\rho_{2}=\rho_{3}=\rho_{4}$. In addition, if we replace $z$ with $\bar{z}$ we get the same results. Consequently, we have $\Lambda_{\epsilon}(A)=\Lambda_{\epsilon}(A)^{*}=\Lambda_{\epsilon}\left(A^{*}\right)=\Lambda_{\epsilon}\left(A^{*}\right)^{*}$. But $A \neq A^{*}$.

Remark 25. We recall that, if $A$ is a Hermitian matrix, then $\Lambda_{0}(A) \subseteq \mathbb{R}$. For the pseudospectrum, we may have $A^{*}=A$, and not have $\Lambda_{\epsilon}(A) \subseteq \mathbb{R}$. Let

$$
A=\left[\begin{array}{cc}
0 & i \\
-i & 0
\end{array}\right], \epsilon=1
$$

and take the Frobenius norm. $\Lambda_{1}(A)=\left\{z \in \mathbb{C}:\left\|(A-z I)^{-1}\right\|_{F} \geq 1\right\}$,

$$
\left\|(A-z I)^{-1}\right\|_{F}=\left\|\left(\begin{array}{cc}
-z & i \\
-i & -z
\end{array}\right)^{-1}\right\|_{F}=\frac{1}{\left|z^{2}-1\right|} \sqrt{2|z|^{2}+2} .
$$

Evidently, there exist a complex number

$$
i \in \Lambda_{1}(A)=\left\{z \in \mathbb{C}: \frac{1}{\left|z^{2}-1\right|} \sqrt{2|z|^{2}+2} \geq 1\right\}
$$

In view of considering realistic situations, the notion of a structured pseudospectrum appears to be useful: If $A$ has some "structural property" it may happen that in practice all perturbations on $A$ belong to the same family.

Theorem 26. If $A^{*}=A$, then $\Lambda_{\epsilon}^{\mathrm{He}}(A) \subseteq \mathbb{R}$.

Proof. Let $z \in \Lambda_{\epsilon}^{\mathrm{He}}(A)$. That is, there existe a hermitian matrix $E \in \mathbb{F}^{n \times n}$ such that $z \in \Lambda_{0}(A+E)$. Since $A=A^{*}$ so $z \in \Lambda_{0}(A+E) \subseteq \mathbb{R}$. 


\subsection{Properties of $\Lambda_{\epsilon}(A)$ with Respect to $\epsilon$}

We showed that each component of $\Lambda_{\epsilon}(A)$ contains at least one eigenvalue of $A$ in its interior. Let $\Delta_{\lambda}$ be a component of $\Lambda_{\epsilon}(A)$ containing $\lambda \in \Lambda_{0}(A)$. Then each point in $\Delta_{\lambda}$ is an $\epsilon$-successor of $\lambda$. In other words, $\Delta_{\lambda}$ is the $\epsilon$-domain of influence of $\lambda$. As $\epsilon$ increases gradually the component $\Delta_{\lambda}$ increases in size and intersects another component $\Delta_{\mu}$ containing an eigenvalue $\mu \in \Lambda_{0}(A)$. Once the intersection takes place the components $\Delta_{\lambda}$ and $\Delta_{\mu}$ lose their identity and form a bigger $\Delta_{\lambda, \mu}$ containing both the eigenvalues $\lambda$ and $\mu$. Thus, by increasing the value of $\epsilon$ gradually the concept of successor of two eigenvalues gets blurred because we have some points of $\Delta_{\lambda, \mu}$ which are successors of both $\lambda$ and $\mu$. Although, it may be difficult to determine the points in $\Delta_{\lambda, \mu}$ which are successors of both $\lambda$ and $\mu$, if the value of $\epsilon$ is such that $\Delta_{\lambda}$ and $\Delta_{\mu}$ just meet then the set of points in $\Delta_{\lambda, \mu}$ which forms the common boundary of $\Delta_{\lambda}$ and $\Delta_{\mu}$ is precisely the set of common successors of $\lambda$ and $\mu$. Futher, when $\Delta_{\lambda}$ intersects more than one component of $\Lambda_{\epsilon}(A)$ the resulting component may be multiply connected. To illustrate these facts we consider a simple example.

Example 1. [5] Let $A=\operatorname{diag}(1,-1, i,-i)$. Since $A$ is diagonal, for small $\epsilon$ the components of $\Lambda_{\epsilon}(A)$ consist of four circles centerd at the eigenvalues of $A$. Figure 1 shows $\Lambda_{\epsilon}(A)$. The eigenvalues of $A$ are shown with $\times$. The innermost

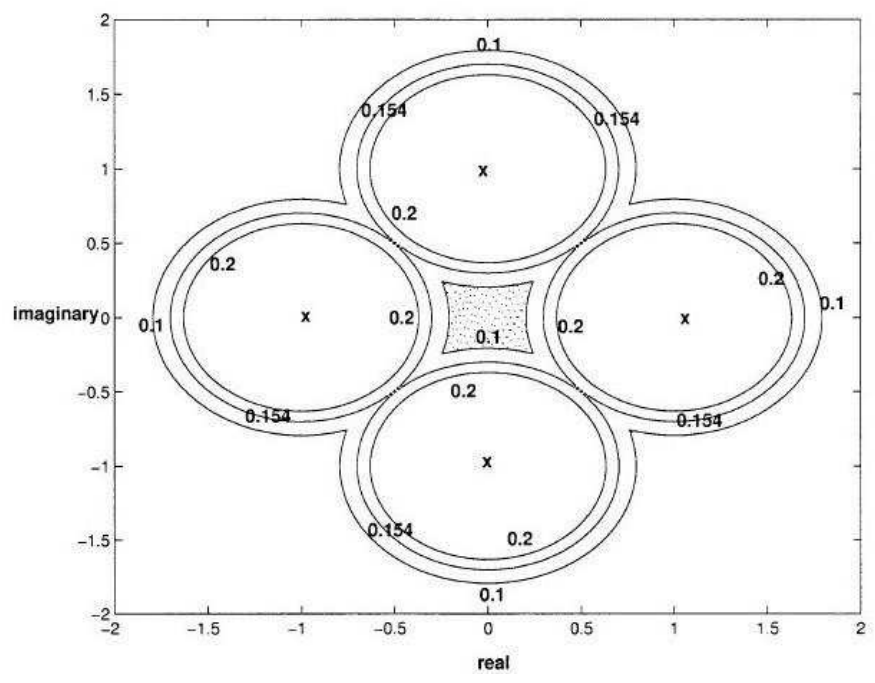

Figure 1: Contour plot of $\Lambda_{\epsilon}(A)$ in $\log _{10}$-scale for $\epsilon=0.63,0.7,0.79$.

contour enclosing the eigenvalues corresponds to $\epsilon=0.63$. The regions enclosed 
by these contours are components of $\Lambda_{\epsilon}(A)$. Thus, for $\epsilon=0.63$ the pseudospectrum $\Lambda_{\epsilon}(A)$ consists of four components and each point in these components is an $\epsilon$-successor of an eigenvalue.

As $\epsilon$ increases the components of $\Lambda_{\epsilon}(A)$ increase in size and coalesce for $\epsilon=0.70$ to form a single multiply connected component. Thus $\Lambda_{\epsilon}(A)$ consists of a single component. The common successors, for example, of the eigenvalues 1 and $i$ are precisely the common points of the contours containing the eigenvalues 1 and i in their interior. Similarly, for other eigenvalues the common successors are given by the common points of the respective contours that enclose them.

When $\epsilon=0.79$, the common boundaries get blurred and hence the common successors too. In this case $\Lambda_{\epsilon}(A)$ consists of a single multiply connected component which is the region enclosed by the outer contours excluding the shaded region.

Definition 27. Let $f: \epsilon \in \mathbb{R}_{+} \mapsto f(\epsilon)$. $f$ is called monotone increasing iff for all $\epsilon_{1}, \epsilon_{2} \in \mathbb{R}_{+}, \epsilon_{1}<\epsilon_{2}$ implies $f\left(\epsilon_{1}\right) \subseteq f\left(\epsilon_{2}\right)$.

Proposition 28. Let $f: \epsilon \in \mathbb{R}_{+} \mapsto \Lambda_{\epsilon}(A) \in P(\mathbb{C}), f$ is monotone increasing and continuous.

Proof. We will follow the lines of [5, Proposition (3.3.3)].

It is easy to see that $f$ is monotone increasing. To prove the continuity of $f$, we show that it is both upper and lower semicontinous. Let $\left(\epsilon_{n}\right)_{n \geq 1}$ be a sequence converging to $\epsilon$.

To prove the upper semicontinuity of $f$, let $G$ be an open subset of $\mathbb{C}$ such that $\Lambda_{\epsilon}(A) \subseteq G$. Then $\|R(A, z)\|<\frac{1}{\epsilon}$ for all $z \in G^{c}$. Since $\|R(A, z)\|$ is subharmonic on the closure of $G^{c}$, therefore, unless $\|R(A, z)\|$ is constant on $G^{c}$, there exists a point $z_{0}$ on the boundary of $G$ such that $\|R(A, z)\|<\left\|R\left(A, z_{0}\right)\right\|$ for all $z \in G^{c}$. If $\|R(A, z)\|$ is constant on $G^{c}$, then $\|R(A, z)\|=0$ for $z \in \mathbb{C}$. Consequently, $\frac{1}{\epsilon}<0$ which is absurd. Thus $\|R(A, z)\|$ is not constant on $G^{c}$ by Liouville's theorem. We can assume that there exists $z_{0} \in \partial G$, the boundary of $G$, such that $\|R(A, z)\|<\left\|R\left(A, z_{0}\right)\right\|$ for all $z \in G^{c}$. Since $\epsilon>0$, it follows that $\frac{1}{\epsilon_{n}} \rightarrow \frac{1}{\epsilon}$ as $n \rightarrow \infty$. Taking $\delta=\frac{1}{\epsilon}-\left\|R\left(A, z_{0}\right)\right\|$, for all large $n$, we have

$$
\frac{1}{\epsilon}-\frac{1}{\epsilon_{n}}<\delta \Longrightarrow\left\|R\left(A, z_{0}\right)\right\|<\frac{1}{\epsilon_{n}} .
$$

Since $\|R(A, z)\|<\left\|R\left(A, z_{0}\right)\right\|$ for all $z \in G^{c}$, it follows that $G^{c} \subseteq \Lambda_{\epsilon_{n}}(A)^{c}$, i.e. $\Lambda_{\epsilon_{n}}(A) \subseteq G$, for all large $n$. This proves the upper semicontinuity of $\Lambda_{\epsilon}(A)$ as a function of $\epsilon$. 
Let $z_{0} \in \Lambda_{\epsilon}(A)$. If $f$ is lower semicontinous then every open set $G \subseteq \mathbb{C}$ containing $z_{0}$ also contains a point of $\Lambda_{\epsilon_{n}}(A)$ for all sufficently large $n$. If we supposed to the contrary, then there exists an open set $G$ such that $z_{0} \in G$ and a subsequence $\left(\epsilon_{n_{k}}\right)_{k \geq 1}$ of $\left(\epsilon_{n}\right)_{n \geq 1}$ such that $\Lambda_{\epsilon_{n_{k}}}(A) \cap G=\phi$ for all $k \in \mathbb{N}$. Since $\epsilon_{n_{k}} \rightarrow \epsilon_{n}$ as $k \rightarrow \infty$, this implies that $\|R(A, z)\| \leq \frac{1}{\epsilon}$ for all $z \in G$. In particular we have $\left\|R\left(A, z_{0}\right)\right\| \leq \frac{1}{\epsilon}$. Since $z_{0} \in \Lambda_{\epsilon}(A)$, this implies that $\left\|R\left(A, z_{0}\right)\right\|=\frac{1}{\epsilon}$. Therefore $\|R(A, z)\| \leq\left\|R\left(A, z_{0}\right)\right\|$ for all $z \in G$. Since $\|R(A, z)\|$ is subharmonic and bounded above on $G$ then $\|R(A, z)\|$ is constant by Liouville's Theorem. This shows that $\|R(A, z)\|=0$ for all $z \in \mathbb{C}$ which is absurd. Hence $f$ is lower semicontious.

Corollary 29. For any function $f: \mathbb{R}_{+} \rightarrow \mathbb{R}_{+}$and for all $\epsilon>0$ we have only one of the two following cases:

1. $\Lambda_{\epsilon}(A) \subseteq \Lambda_{f(\epsilon)}(A)$ if $f(\epsilon)>\epsilon$,

2. $\Lambda_{f(\epsilon)}(A) \subseteq \Lambda_{\epsilon}(A)$ if $f(\epsilon)<\epsilon$.

We have mentioned the geometric separation of two eigenvalues $\lambda \neq \mu$. The geometric separation of $\Lambda_{1}, \Lambda_{2}, \ldots, \Lambda_{m}$ is defined by

$$
\begin{aligned}
\operatorname{gsep}\left(\Lambda_{1}, \ldots, \Lambda_{m}, A\right)= & \min \left\{\epsilon>0: \Lambda_{\epsilon}(A)\right. \text { has a component which } \\
& \text { intersects } \left.\Lambda_{i} \text { and } \Lambda_{j} \text { for some } i \neq j\right\} .
\end{aligned}
$$

We denote the geometric separation of a subset $\Lambda$ of $\Lambda_{0}(A)$ from the rest of $\Lambda_{0}(A)$ by $\operatorname{gsep}(\Lambda)$, and for an eigenvalue by $\operatorname{gsep}(\lambda)$.

Proposition 30. Let $\Lambda \subseteq \Lambda_{0}(A)$. Then $\Lambda_{\epsilon}(A)$ has components which together isolate $\Lambda$ from the rest of $\Lambda_{0}(A)$ iff $\epsilon<\operatorname{gsep}(\Lambda)$.

Properties of the critical dissociation and geometric separation of two eigenvalues $\lambda \neq \mu$ are found in [5].

\section{References}

[1] M. Ahues, Cours de Théorie Spectrale, polycopié Master 1 de Mathématiques à Saint-Étienne (2005).

[2] M. Ahues and N. Vernay, Approximation spectrale d'opérateurs linéaires, polycopié Master 2 Modélisation Mathématique à Saint-Étienne (2006). 
[3] R. Alam and S. Bora, On pseudospectral analysis of matrices, International Workship On Linear Algebra, Numerical Functional Analysis And Wavelet Analysis (2001), 25-44.

[4] R. Alam and S. Bora, On sensitivity of eigenvalues and eigendecompositions of matrices, Linear Algebra and its Applications, 396 (2005), 273-301.

[5] S. Bora, A geometric analysis of spectral stability of matrices and operators, Ph.D thesis, IIT Guwahati, India (2001).

[6] J.W. Demmel, Computing stable eigendecompositions of matrices, Linear Algebra and its Applications, 79, (1986), 163-193.

[7] N.J. Higham, Accuracy and stability of Numerical Algorithms, SIAM Publications, Philadelphia, 2nd edition (2002).

[8] R.A. Horn and Ch. Johnson, Matrix Analysis, Cambridge University Press (1985).

[9] S.M. Rump, Eigenvalues, pseudospectrum and structured perturbations, Linear Algebra and its Applications, 413 (2006), 567-593.

[10] G.W. Stewart, Error and perturbation bounds for subspaces associated with certain eigenvalue problems, SIAM Review, 15,4 (1973), 727-764.

[11] L.N. Trefethen, Pseudospectra of Linear Operator, SIAM Review, 39,3 (1997), 384-406.

[12] L.N. Trefethen, Pseudospectra of matrices, In: D.F. Griffiths and G.A. Waston, Numerical Analysis, Longman, (1992), 234-266.

[13] J.H. Varah, On the separation of two matrices, SIAM J. Numer. Anal., 16,2 (1979), 216-222.

[14] J.H. Wilkinson, The Algebraic Eigenvalue Problem, Oxford University Press (1966).

[15] J.H. Wilkinson, Sensitivity of eigenvalues, Utilitas Mathematica, 25 (1984), 5-76.

[16] J.H. Wilkinson, Sensitivity of eigenvalues II, Utilitas Mathematica, 30 (1986), 243-286. 